Canadian Oncology

Nursing Journal

Revue canadienne

de soins infirmiers

en oncologie

Volume 28, Issue 2 • Spring 2018

elSSN: 2368-8076 


\title{
Sens et motivation dans les soins infirmiers en oncologie auprès des personnes âgées : de la conférence au groupe d'intérêt
}

\author{
par Fay J. Strohschein et Lorelei J. Newton
}

\section{RÉSUMÉ}

Au Canada, $45 \%$ des nouveaux cas de cancer et $63 \%$ des décès dus au cancer se produisent chez les Canadiens de 70 ans et plus. Ces personnes âgées et leurs familles ont des préoccupations et des besoins particuliers, mais qui demeurent souvent mal reconnus et satisfaits. Or, le nombre de personnes âgées au Canada devrait plus que doubler au cours des 25 prochaines années. Nous devons donc intégrer à notre pratique en oncologie, de même qu'aux politiques, à la recherche et à la formation, une meilleure compréhension du vieillissement, et développer des modèles de soins optimisés et adéquats pour les personnes âgées.

Le groupe d'intérêt sur l'oncologie et le vieillissement de l'Association canadienne des infirmières en oncologie (ACIO) est une initiative incitant les infirmières du domaine à se pencher sur ces questions. Nous résumons ici les faits saillants de l'atelier qui a donné le coup d'envoi à ce groupe lors de la conférence annuelle de l'ACIO en 2015 et rappelons les priorités et problèmes liés à la pratique qui ont été relevés pendant les échanges avec les participants. Nous relatons également la façon dont le groupe d'intérêt a évolué depuis sa création et listons les objectifs qui orienteront les prochaines étapes.

\section{INTRODUCTION}

T 'impact du vieillissement de la population canadienne ـur la prestation des soins de santé se remarque de plus en plus, particulièrement dans les soins contre le cancer. On estime que $45 \%$ des nouveaux cas de cancer et $63 \%$ des décès dus au cancer se produiront chez les Canadiens de 70 ans et plus, et la majorité des cancers les plus fréquents (poumon,

\section{AU SUJET DES AUTEURES}
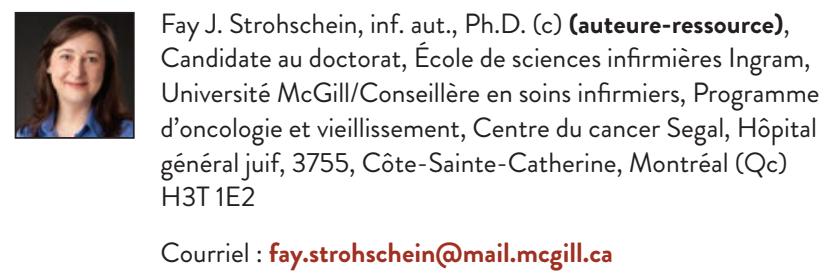

Fay J. Strohschein, inf. aut., Ph.D. (c) (auteure-ressource), Candidate au doctorat, École de sciences infirmières Ingram, Université McGill/Conseillère en soins infirmiers, Programme d'oncologie et vieillissement, Centre du cancer Segal, Hôpital général juif, 3755, Côte-Sainte-Catherine, Montréal (Qc) H3T 1E2

Courriel : fay.strohschein@mail.mcgill.ca

Lorelei J. Newton, inf. aut., Ph.D., Professeure, Nursing, Health and Human Services, Camosun College, 3100 Foul Bay Road, Victoria (C.-B.) V8P 5J2

Courriel : NewtonL@camosun.ca

DOI:10.5737/2368807628295101 colorectal) se produisent dans cette tranche d'âge (Comité consultatif de la Société canadienne du cancer, 2017). Divers signes attestent de lacunes liées à l'âge dans les soins oncologiques : disparités dans les taux de mortalité des différents groupes d'âge (Comité consultatif de la Société canadienne du cancer, 2017), écart grandissant avec l'âge quant au taux de survie (Brenner et Arndt, 2004), besoins insatisfaits (Puts, Papoutsis, Springall et Tourangeau, 2012), tendances de traitements sous-optimaux même lorsque l'état de santé et l'état fonctionnel sont pris en compte (Bojer et Roikjær, 2015; Hamaker et al., 2015).

En tant qu'infirmières en oncologie, nous sommes bien placées pour intégrer aux soins des personnes atteintes de cancer la compréhension des aspects biopsychosociaux du vieillissement. Le travail auprès des personnes âgées a beau représenter une part importante de notre pratique, nous avons souvent peu de formation à cet égard, et un accès limité aux ressources gériatriques. Tenons-nous vraiment compte des besoins particuliers qui relèvent du vieillissement, ou nous adonnons-nous simplement à soigner des gens qui se trouvent dans cette tranche d'âge? Chose certaine, au Canada, le nombre de personnes âgées devrait plus que doubler au cours des 25 prochaines années (Statistique Canada, 2014), et il en ira de même pour le nombre de cas de cancer (Comité consultatif de la Société canadienne du cancer, 2015). Nous devrons donc nous doter des connaissances, compétences et ressources nécessaires pour répondre aux besoins des patients âgés et de leurs proches.

Pour entamer une discussion sur la façon dont les infirmières en oncologie peuvent mieux prendre soin des personnes âgées atteintes de cancer, nous avons organisé un atelier à la conférence annuelle de l'Association canadienne des infirmières en oncologie (ACIO) en octobre 2015. Un groupe d'intérêt sur l'oncologie et le vieillissement en est né. Le présent article relate les grandes lignes de cet atelier et les premières activités du groupe d'intérêt.

Le document s'articule autour des trois grands thèmes de la conférence de 2015 : communauté, volonté, passion, à quoi nous avons ajouté progrès. Nous résumons d'abord les propos tenus à l'atelier en relevant les principales préoccupations en lien avec les soins oncologiques prodigués aux personnes âgées (aspect communauté) et en présentant la volonté qui anime l'oncologie gériatrique en tant que sous-spécialité. Nous rapportons ensuite les conversations des participants à l'atelier, témoignages d'une passion évidente à l'idée de mieux comprendre et aborder les questions relatives au vieillissement des patients. Les participants avaient alors lancé l'idée du groupe d'intérêt et discuté des priorités qu'il devrait avoir. 
Nous évaluons enfin les progrès que le groupe d'intérêt a réalisés et listons les buts et objectifs qui orienteront les prochaines étapes.

\section{COMMUNAUTÉ : LES PROBLÈMES PARTICULIERS AUX SOINS DES PERSONNES ÂGÉES ATTEINTES DU CANCER}

Les façons dont les personnes âgées font l'expérience du cancer et des traitements corollaires varient énormément. Ces expériences sont façonnées par l'hétérogénéité des aspects biologiques, psychologiques et sociaux du vieillissement. Or, ces dissemblances ne sont pas seulement évidentes lorsqu'on compare deux personnes différentes : elles se manifestent quand on compare différentes générations (Gubrium et Charmaz, 1992) et même, lorsqu'on compare différents systèmes d'organes chez un même un individu (White et Cohen, 2006).

Quand on pense à la population « gériatrique », on imagine parfois des aînés fragiles. Mais ces derniers ne représentent qu'un petit sous-ensemble des personnes âgées recevant des traitements contre le cancer (Retornaz et al., 2008). Une donnée éloquente : l'espérance de vie moyenne des femmes de 80 ans peut varier de 13,0 ans, pour celles dans les 25 centiles supérieurs, à 4,6 ans pour celles dans le quart inférieur (Walter et Covinsky, 2001). Ainsi une dame peut-elle être confinée à un fauteuil roulant et requérir une assistance au quotidien, alors qu'une autre peut vivre seule, faire du sport tous les jours et s'impliquer activement dans sa communauté. L’âge chronologique ne suffit donc pas à déterminer les préoccupations et besoins réels d'une personne. Plutôt que de simplement qualifier un patient « d'âgé » en comptant ses années, il nous faut, dans le traitement du cancer, tenir compte de l'âge fonctionnel (Klepin, Rodinet Hurria, 2015) ou de l'âge physiologique (National Cancer Institute, 2010), et nous sensibiliser aux problèmes associés à la vieillesse qui peuvent se manifester à différents moments chez différentes personnes.

La pathologie du cancer change avec l'âge; certains cancers deviennent plus agressifs, et d'autres, indolents (White et Cohen, 2006). D'autres changements physiologiques normaux ou anormaux se produisent au fil des années, comme l'affaiblissement de l'adaptabilité et du maintien de l'homéostasie. L'ampleur de ces changements n'est pas toujours prévisible, et peut avoir de profondes répercussions sur l'efficacité du traitement, de même que sur la capacité du patient à adhérer à un plan de traitement (Sawhney, Sehlet Naeim, 2005; Sehl, Sawhneyet Naeim, 2005). Les changements physiologiques peuvent aussi entraîner une réduction de la mobilité, des troubles sensoriels ou cognitifs, des pathologies multiples, ou l'occurrence de facteurs de risques importants pour les syndromes gériatriques tels que le déclin fonctionnel, la polypharmacie, les chutes, le delirium, l'incontinence, la perte de poids et la dépression (Inouye, Studenski, Tinettiet Kuchel, 2007; Olde Rikkert, Rigaud, van Hoeyweghenet de Graaf, 2003). La variation de la situation sociale d'un individu peut le rendre plus ou moins vulnérable au risque (Andrew et Keefe, 2014), ce qui souligne la nécessité de comprendre le point de vue du patient et d'adapter les soins à la situation de chaque personne.

Le manque de sensibilisation à l'hétérogénéité et aux problèmes associés au vieillissement mène à l'âgisme - à des stéréotypes à propos du déclin et à des idées reçues sur ce que serait un « vieillissement réussi ». Lâgisme est insidieux dans le système de santé (Alliance for Aging Research, 2003) et l'oncologie ne fait pas exception (Kagan, 2008; Wedding et Stauder, 2014). Même si les formes de discrimination les plus flagrantes sont souvent évitées, les plus subtiles sont communément ignorées. Parmi les exemples, mentionnons la présentation inadéquate de l'information, l'inaccessibilité des milieux de soins de santé, le manque de coordination des soins, les ressources limitées pour assurer l'accès des patients âgés au dépistage et au traitement, et des variations dans la gestion clinique en fonction des valeurs et des perceptions de l'équipe soignante en oncologie (Tariman, 2009; Tariman, Berry, Cochrane, Doorenboset Schepp, 2012). La perpétuation de ces schémas d'âgisme vient notamment $\mathrm{du}$ manque de recherches sur cette population, notamment en raison du peu d'inclusion dans les essais cliniques et d'un nombre insuffisant de mesures des résultats reflétant les paramètres importants pour les personnes âgées (Hurria et al., 2014; Hurria et al., 2015; Sinding et Wiernikowski, 2009). Il faut corriger le tir pour fournir des traitements adéquats et offrir aux aînés les soins dont ils ont vraiment besoin.

\section{VOLONTÉ : LE RÔLE DE L'ONCOLOGIE GÉRIATRIQUE}

Sous-spécialité qui prend de l'ampleur, l'oncologie gériatrique adjoint l'étude du vieillissement à l'oncologie en vue d'offrir aux personnes âgées atteintes de cancer et à leurs proches des soins optimaux et adaptés. C'est une discipline relativement récente. Les préjugés dans le traitement des personnes âgées ont été reconnus pour la première fois au milieu des années 1970-1980; après une période de revendication, des avancées significatives ont commencé à se produire dans les années 1990 (Boyle, 2006). L’Oncology Nursing Society a publié son premier énoncé de position sur l'oncologie gériatrique et les soins infirmiers en oncologie en 1992 (Boyle et al., 1992); il a ensuite été mis à jour en collaboration avec le Geriatric Oncology Consortium (Oncology Nursing Society et Geriatric Oncology Consortium, 2004, 2007). Cet énoncé évoque le manque de recherche et la complexité des soins qui transparaissent dans les disparités et tendances au sous-traitement qui ont une incidence sur la qualité de vie. Dans un rapport récent sur les soins de qualité en cancérologie, l'Institute of Medicine souligne l'importance de la collaboration entre les équipes primaires, gériatriques et spécialisées (Institute of Medicine, 2013), la coopération entre les infirmières en oncologie et les infirmières gériatriques étant indispensable à l'atteinte de cet objectif (Ferrell, McCabeet Levit, 2013).

Pour garantir des soins adéquats, l'oncologie gériatrique doit reposer sur une collaboration interdisciplinaire et interprofessionnelle. De nombreuses personnes âgées qui arrivent 
dans un centre de cancérologie ont l'air fortes et indépendantes; il se peut toutefois qu'elles soient vulnérables aux effets toxiques du traitement, ou à d'autres complications ou effets secondaires que l'équipe ne peut soupçonner lors des premières rencontres. Une évaluation gériatrique complète permet de déterminer les forces et faiblesses du patient au départ, d'en évaluer le statut fonctionnel, et de détecter des vulnérabilités aux complications, aux effets secondaires ou à la toxicité (National Comprehensive Cancer Network, 2016; Wildiers et al., 2014). À l'instar de la communauté médicale qui se mobilise pour soutenir le développement de l'oncologie gériatrique en tant que sous-spécialité dans la recherche, l'éducation, la pratique et l'élaboration des politiques, la communauté infirmière doit s'activer au sein de sa propre profession. Nous devons tenir compte des compétences relatives au vieillissement que requiert la prestation de soins efficaces, et nous devons favoriser le perfectionnement professionnel des infirmières qui œuvrent dans cette sous-spécialité.

\section{PASSION : LES DÉFIS ET PRÉOCCUPATIONS DANS LES SOINS INFIRMIERS}

Ayant constaté notre intérêt commun pour ces sujets, nous (les auteures du présent article) avons jugé nécessaire d'échanger avec d'autres infirmières en oncologie. Pour entamer le dialogue et explorer la possibilité de former un groupe d'intérêt, nous avons organisé à la conférence annuelle de l'ACIO de 2015 un atelier ouvert à tous les participants. En mettant en commun notre expérience (Fay était alors infirmière clinicienne spécialisée et infirmière pivot pour les patients âgés en oncologie au Centre du cancer Segal à Montréal, et Lorelei, responsable de la pratique pour la British Columbia Cancer Agency à Victoria), nous avons donné trois objectifs à l'atelier :

- Faire un survol des façons dont l'oncologie et le vieillissement s'entrecroisent, et considérer les besoins afférents qui ne sont pas assez reconnus et étudiés.

- Faire ressortir la raison d'être de l'oncologie gériatrique en tant que sous-spécialité.

- Animer une discussion avec les participants pour explorer les défis et les bons coups réalisés auprès des personnes âgées atteintes de cancer, et parler de la création d'un groupe d'intérêt.

Douze collègues travaillant dans différents contextes cliniques à travers le pays ont participé à l'atelier. Ensemble, nous avons commencé à explorer les enjeux aussi bien que les réussites. Les participants ont fait des témoignages prenants, évoquant toute la complexité qu'implique le fait de prendre soin de personnes âgées atteintes de cancer tout au long de la trajectoire de soins... Leur passion est évidente. Mais il est aussi apparu que les infirmières en oncologie vivent de la détresse morale quand les ressources et les systèmes requis pour répondre à ces besoins ne sont pas en place.

Cette détresse morale émanait en particulier des histoires de sous-traitements et de traitements trop agressifs. Des questions ont alors fait surface... Bien que la plupart des personnes âgées soient plutôt en bonne santé quand elles entament un traitement, dans quel état les laissons-nous à la fin? Les aidonsnous à retrouver le niveau de fonctionnement qu'ils avaient au départ? Savons-nous seulement ce qu'il était? À quoi ressemblerait l'intégration d'une approche gérontologique à nos évaluations et interventions tout au long de la trajectoire de soins? Quand et comment aiguiller adéquatement les personnes âgées vers les soins palliatifs? Nous nous sommes également penchés sur les questions systémiques, en discutant des décisions explicites et implicites concernant l'accès au traitement et son rationnement, et la façon dont l'âge du patient peut influencer ces décisions.

$\mathrm{Au}$ fil de la discussion, nous avons mis au jour de nombreuses préoccupations cliniques liées aux soins oncologiques pour les personnes âgées. Les participants ont verbalisé trois grandes sources d'inquiétudes (tableau 1) : l'âgisme, les résultats des traitements sous-optimaux, et les questions éthiques concernant les décisions en matière de soins et de traitements. Les participants ont non seulement exprimé des préoccupations précises en lien avec ces trois sujets, mais ils ont aussi proposé des objectifs et des idées sur la façon dont le système de soins contre le cancer pourrait y répondre.

Le tableau 1 recense les préoccupations des infirmières en oncologie à l'égard du vieillissement, et les changements proposés au système. Les participants ont également reconnu que les déterminants sociaux de la santé - tels que le revenu, l'éducation, l'exclusion sociale, le sexe et la race - interagissent avec les enjeux cernés, en ayant une incidence sur les ressources et en accroissant les besoins et les inquiétudes. Cette interaction serait particulièrement marquée au sein de certaines populations cancéreuses, comme celles atteintes d'un cancer de la tête et du cou ou d'un myélome multiple, et celles vivant en milieu rural.

Comme nous l'avons mentionné, l'atelier a permis de comprendre que l'écart entre les besoins des personnes âgées atteintes de cancer et les réponses du système était la cause de la détresse morale chez nos participants. Nous nous sommes accordés sur le fait que l'âgisme n'a pas qu'une incidence directe sur les personnes âgées et sur leur capacité à recevoir des traitements optimaux et adéquats : cette discrimination entrave aussi notre propre capacité à changer le système pour mieux aider les patients et leur famille. Cela est, en soi, une importante source de frustration. Ensemble, tâchons de nous pourvoir des ressources, des outils, des compétences et des connaissances nécessaires pour satisfaire ces besoins. Pour y parvenir, nous devrons travailler à différents niveaux : éducation, pratique, recherche et politiques.

Le groupe a reconnu qu'une première étape cruciale consistait à sensibiliser les membres de l'ACIO à ces sujets et à rallier les infirmières qui s'intéressent au traitement du cancer chez les aînés. Pour donner le coup d'envoi à ce travail collaboratif, nous avons convenu de fonder un groupe d'intérêt sur l'oncologie et le vieillissement au sein de l'ACIO, une initiative conforme au plan stratégique de l'association, qui met à notre disposition des outils et ressources. Nous avons conclu l'atelier en discutant des priorités qu'aurait ce groupe (résumées dans le tableau 2). 


\begin{tabular}{|c|c|c|}
\hline Âgisme & $\begin{array}{l}\text { Concentration sur l'âge chronologique sans prise en compte des } \\
\text { capacités fonctionnelles et des comorbidités. } \\
\text { Vieillesse vue comme une comorbidité plutôt que comme une } \\
\text { étape du développement humain. } \\
\text { Personnes âgées considérées comme des citoyens de seconde } \\
\text { classe. } \\
\text { Absence d'évaluations gériatriques complètes pour tous les } \\
\text { patients. } \\
\text { Difficulté à reconnaître que la prochaine génération puisse être } \\
\text { différente. }\end{array}$ & $\begin{array}{l}\text { Établir des liens avec la gériatrie pour faciliter des évaluations complètes; } \\
\text { reconnaître l'hétérogénéité et identifier les besoins associés au } \\
\text { vieillissement. } \\
\text { Faire de la recherche pour soutenir l'implication des praticiens en gériatrie. } \\
\text { Donner accès aux services d'une infirmière clinicienne spécialisée en } \\
\text { gériatrie. } \\
\text { Intégrer des processus pour prioriser les patients âgés en vue d'effectuer } \\
\text { une évaluation gériatrique complète. } \\
\text { Mettre au point des outils pour aider les personnes âgées qui n'ont pas } \\
\text { accès à une évaluation gériatrique complète ou qui n'en ont pas besoin. } \\
\text { Apporter une perspective gérontologique aux soins, y compris les soins } \\
\text { palliatifs. }\end{array}$ \\
\hline $\begin{array}{l}\text { Problèmes } \\
\text { éthiques } \\
\text { concernant } \\
\text { les décisions } \\
\text { relatives aux } \\
\text { soins et au } \\
\text { traitement }\end{array}$ & $\begin{array}{l}\text { Besoin de reconnaître que les patients âgés ont souvent une } \\
\text { confiance presque aveugle en leurs médecins traitants. } \\
\text { Explications inadéquates et trop peu claires quant aux } \\
\text { traitements, ce qui amène les patients à subir des traitements } \\
\text { qu'ils ne comprennent pas pleinement. } \\
\text { Difficultés à protéger l'autodétermination et la sécurité des } \\
\text { patients. }\end{array}$ & $\begin{array}{l}\text { Militer pour un traitement et un soutien à la décision adéquats pour les } \\
\text { patients. } \\
\text { Veiller à ce que les patients âgés recevant des soins primaires soient } \\
\text { recommandés à des spécialistes en oncologie. } \\
\text { Insister sur la capacité des personnes âgées à prendre des décisions } \\
\text { concernant leur traitement. } \\
\text { Optimiser les façons de soutenir l'indépendance en dépit des risques, tout } \\
\text { en mettant en place les mesures de soutien nécessaires. }\end{array}$ \\
\hline
\end{tabular}

\section{PROGRÈS : LES PRIORITÉS ET LES OBJECTIFS DU GROUPE D'INTÉRÊT}

L'atelier de 2015 a souligné l'intérêt pour un groupe sur l'oncologie et le vieillissement au sein de l'ACIO; ce dernier a donc été créé dès décembre de la même année. Notre première rencontre annuelle s'est tenue en 2016 à la conférence de l'ACIO. Nous y avons discuté des priorités du groupe et relevé différents besoins : intégrer au programme de la conférence davantage d'activités en lien avec le traitement des personnes âgées; préparer des webinaires ou ateliers portant sur des outils et ressources à l'attention des infirmières en oncologie travaillant auprès des personnes âgées et de leurs familles; préciser le rôle des infirmières en oncologie dans le contexte de l'évaluation clinique des personnes âgées atteintes de cancer. Cette discussion s'est poursuivie dans les rencontres subséquentes, et nous continuons de définir les prochaines étapes et notre plan stratégique global. Sur la base de ces échanges, et conformément aux priorités identifiées par les participants et les membres du groupe, nous avons défini l'objet, le but et les objectifs du groupe d'intérêt (tableau 3). L'objet et les objectifs initiaux ont été approuvés par les membres du groupe en mai 2017. 
Tableau 2 : Priorités du groupe d'intérêt selon les participants

\begin{tabular}{|c|c|}
\hline Priorités & Idées d'actions concrètes \\
\hline $\begin{array}{l}\text { Une première étape } \\
\text { cruciale : sensibiliser }\end{array}$ & $\begin{array}{l}\text { - Informer les infirmières en oncologie des } \\
\text { enjeux : } \\
\text { - les prévisions statistiques : une } \\
\text { augmentation spectaculaire du nombre } \\
\text { de Canadiens âgés atteints de cancer; } \\
\text { - les impacts que cela risque d'avoir sur } \\
\text { la prestation des soins. } \\
\text { - Mieux faire connaître les enjeux associés } \\
\text { au vieillissement et risquant d'avoir un } \\
\text { effet sur les soins apportés aux patients } \\
\text { atteints du cancer et sur leurs familles. }\end{array}$ \\
\hline $\begin{array}{l}\text { Mettre au point des } \\
\text { outils pour mobiliser } \\
\text { les membres et faire } \\
\text { croître le groupe } \\
\text { d'intérêt }\end{array}$ & $\begin{array}{l}\text { - Créer un site Web où rassembler des } \\
\text { ressources. } \\
\text { - Utiliser Listserv pour les membres. }\end{array}$ \\
\hline $\begin{array}{l}\text { Identifier un projet } \\
\text { tangible sur lequel } \\
\text { travailler ensemble } \\
\text { pour faire progresser } \\
\text { la pratique }\end{array}$ & $\begin{array}{l}\text { - Sonder les membres de l'ACIO sur } \\
\text { les besoins relatifs au traitement des } \\
\text { personnes âgées. } \\
\text { - Développer un outil d'évaluation } \\
\text { gériatrique normalisé pour les infirmières } \\
\text { en oncologie. Cet outil devrait : } \\
\text { - faciliter le dépistage menant à une } \\
\text { évaluation gériatrique complète; } \\
\text { - aider à identifier les déficits sensoriels } \\
\text { et cognitifs qui peuvent avoir une } \\
\text { incidence sur la communication; } \\
\text { - tenir compte des valeurs, priorités, } \\
\text { objectifs et forces du patient et de sa } \\
\text { famille. }\end{array}$ \\
\hline
\end{tabular}

Pour soutenir nos priorités et nos objectifs initiaux, nous avons conçu un site pour les membres du groupe d'intérêts accessible par la plateforme Web de l'ACIO. Il permet d'accéder à des ressources communes et à un forum où les membres peuvent échanger et poser des questions. Nous préparons aussi des webinaires et des conférences visant à sensibiliser les membres de l'ACIO à ces sujets. Nous tissons également des liens avec des organisations nationales et internationales soucieuses des soins fournis aux aînés atteints de cancer, comme l'Association canadienne des infirmières et des infirmiers en gérontologie, le Canadian Network on Aging and Cancer, et le groupe d'intérêt des infirmiers et autres professionnels de la santé de la Société internationale d'oncologie gériatrique. En decembre 2017, nous avions 99 membres de partout au Canada et aux États-Unis qui représentaient divers domaines de la pratique clinique, dont des infirmières de chevet, des infirmières cliniciennes, des infirmières pivots, des infirmières en pratique avancée, des chercheurs en sciences infirmières, des coordonnateurs de recherche, des éducateurs et des étudiants.
Tableau 3 : Objet et objectifs du groupe d'intérêt sur l'oncologie et le vieillissement

Objet : Créer un forum national pour les infirmières qui partagent un intérêt pour les soins aux personnes âgées atteintes de cancer, et s'impliquer à la grandeur du Canada pour promouvoir l'innovation dans la pratique, la recherche, l'éducation, le leadership et l'élaboration de politiques.

But : Conjuguer les connaissances sur l'oncologie et sur le vieillissement pour optimiser les soins offerts aux personnes âgées atteintes de cancer et le soutien de leurs proches.

\section{Objectifs :}

Sensibilisation du milieu

- Préconiser des soins optimaux pour les personnes âgées atteintes de cancer (et le soutien aux proches), en attirant l'attention sur leurs forces, besoins et préoccupations particuliers, tant dans le cadre des initiatives de l'ACIO qu'au-delà.

- Souligner le rôle crucial des infirmières au sein des équipes interprofessionnelles et interdisciplinaires dans l'optimisation des soins prodigués aux personnes âgées atteintes de cancer et du soutien accordé à leurs proches.

Mobilisation des membres

- Repérer, élaborer et diffuser des outils et ressources qui favorisent l'essor des compétences et des connaissances relatives au soin des personnes âgées atteintes de cancer et de leurs proches.

Progression de la pratique

- Entreprendre des projets pour améliorer les soins offerts aux personnes âgées atteintes de cancer (et le soutien des proches) en partant des enjeux, tendances et besoins cernés par nos membres.

- Promouvoir les soins infirmiers en oncologie gériatrique en tant que sous-spécialité visant à intégrer les expertises en gériatrie et dans les soins du cancer et en définir les normes et les compétences requises selon l'ACIO.

Accent mis sur la collaboration

- Collaborer avec d'autres organisations nationales et internationales qui visent à améliorer les soins offerts aux personnes âgées atteintes de cancer.

\section{CONCLUSION}

Intégrer la compréhension du vieillissement aux soins en cancérologie est en train de devenir une préoccupation importante pour les infirmières en oncologie. Notre atelier a confirmé que ces dernières se soucient énormément de la qualité des soins offerts aux personnes âgées. Les participants ont parlé des façons dont les infirmières en oncologie pouvaient combattre l'âgisme, en reconnaissant l'hétérogénéité et les enjeux relatifs au vieillissement, en se dotant des outils et ressources nécessaires pour optimiser les résultats des traitements, et en exigeant un meilleur soutien au traitement et à la prise de décision pour s'attaquer aux problèmes éthiques qui s'y rapportent. Ces échanges nous ont vivement encouragées à créer un groupe d'intérêt sur l'oncologie et le vieillissement, et 
en ont orienté les priorités et les buts initiaux. Nous entendons bientôt rédiger notre plan stratégique et comptons aller chercher le point de vue des personnes âgées atteintes du cancer et leurs proches pour l'intégrer à notre démarche.

Grâce à la présence de ce groupe d'intérêt au sein de l'ACIO, nous espérons contribuer à la concrétisation et à l'actualisation d'un modèle de traitement du cancer qui facilite cette intégration, en donnant les bons outils aux infirmières en oncologie qui travaillent avec des patients âgés, et en soutenant le perfectionnement des infirmières spécialisées en oncologie gériatrique. Avec ce groupe d'intérêt, nous espérons attirer l'attention sur les forces, les besoins et les préoccupations

\section{RÉFÉRENCES}

Alliance for Aging Research. (2003). Ageism: How healthcare fails the elderly. Washington, DC. Retrieved from https://www. agingresearch.org/pressrooms/view/51\#.WbwXfLpFyhI

Andrew, M.K., \& Keefe, J.M. (2014). Social vulnerability from a social ecology perspective: a cohort study of older adults from the National Population Health Survey of Canada. BMC Geriatrics, 14(1), 90. doi:10.1186/1471-2318-14-90

Bojer, A.S., \& Roikjær, O. (2015). Elderly patients with colorectal cancer are oncologically undertreated. European Journal of Surgical Oncology, 41(3), 421-425. doi:10.1016/j.ejso.2014.10.065

Boyle, D.A. (2006). A review of the evidence base of an evolving science: Gero-oncology nursing. In H.B. Muss, C.P. Hunter \& K.A. Johnson (Eds.), Treatment and management of cancer in the elderly (pp. 507-538). New York: Taylor \& Francis Group.

Boyle, D.M., Engelking, C., Blesch, K.S., Dodge, J., Sarna, L., \& Weinrich, S. (1992). Oncology Nursing Society position paper on cancer and aging: The mandate for oncology nursing. Oncology Nursing Forum, 19(6), 913-933.

Brenner, H., \& Arndt, V. (2004). Recent increase in cancer survival according to age: Higher survival in all age groups, but widening age gradient. Cancer Causes Q Control, 15(9), 903-910. doi:10.2307/3553599

Canadian Cancer Society's Advisory Committee on Cancer Statistics (2015). Canadian Cancer Statistics 2015. Toronto, ON: Canadian Cancer Society.

Canadian Cancer Society's Advisory Committee on Cancer Statistics (2017). Canadian Cancer Statistics 2017. Toronto, ON: Canadian Cancer Society.

Ferrell, B., McCabe, M.S., \& Levit, L. (2013). The Institute of Medicine report on high-quality cancer care: Implications for oncology nursing. Oncology Nursing Forum, 40(6), 603-609. doi:10.1188/13. onf.603-609

Gubrium, J.F., \& Charmaz, K. (1992). Introduction. In J.F. Gubrium $\&$ K. Charmaz (Eds.), Aging, self, and community: A collection of readings (pp. ix-x). Greenwich, Connecticut: Jai Press.

Hamaker, M.E., van Rixtel, B., Thunnissen, P., Oberndorff, A.H., Smakman, N., \& ten Bokkel Huinink, D. (2015). Multidisciplinary decision-making on chemotherapy for colorectal cancer: An agebased comparison. Journal of Geriatric Oncology, 6(3), 225-232. doi:10.1016/j.jgo.2015.01.002

Hurria, A., Dale, W., Mooney, M., Rowland, J.H., Ballman, K.V., Cohen, H.J., ... Mohile, S.G. (2014). Designing therapeutic clinical trials for older and frail adults with cancer: U13 conference recommendations. Journal of Clinical Oncology, 32(24), 2587-2594. doi:10.1200/JCO.2013.55.0418

Hurria, A., Levit, L.A., Dale, W., Mohile, S.G., Muss, H.B., Fehrenbacher, L., ... Cohen, H.J. (2015). Improving the evidence des personnes âgées atteintes de cancer et de leurs proches, et offrir une tribune nationale qui stimulera l'innovation dans les soins qu'on leur porte.

\section{REMERCIEMENTS}

Nous remercions les participants à l'atelier que nous avons donné à la Conférence annuelle 2015 de l'ACIO pour leur enthousiasme et leurs réflexions, qui ont jeté les bases de ce travail. Nous saluons également ceux qui ont participé aux réunions subséquentes $d u$ groupe d'intérêt : votre apport inestimable nous a aidées à fixer les priorités, les objectifs et les activités du groupe.

base for treating older adults with cancer: American Society of Clinical Oncology Statement. Journal of Clinical Oncology, 33(32), 3826-3833. doi:10.1200/jco.2015.63.0319

Inouye, S.K., Studenski, S., Tinetti, M.E., \& Kuchel, G.A. (2007). Geriatric syndromes: Clinical, research, and policy implications of a core geriatric concept. Journal of the American Geriatrics Society, 55(5), 780-791. doi:10.1111/j.1532-5415.2007.01156.x

Institute of Medicine (2013). Delivering high-quality cancer care: Charting a new course for a system in crisis. Washington, DC: The National Academies Press.

Kagan, S.H. (2008). Ageism in cancer care. Seminars in Oncology Nursing, 24(4), 246-253. doi:10.1016/j.soncn.2008.08.004

Klepin, H.D., Rodin, M., \& Hurria, A. (2015). Treating older adults with cancer: Geriatric perspectives. In D.S. Dizon (Ed.), 2015 American Society of Clinical Oncology Educational Book. Alexandria, VA: American Society of Clinical Oncology.

National Cancer Institute (2010). Focusing on older cancer patients: A clinical need and a research necessity. Retrieved from https://www. cancer.gov/about-cancer/treatment/research/older-patients

National Comprehensive Cancer Network (2016). The NCCN Clinical Practice Guidelines in Oncology (NCCN Guidelines ${ }^{\mathrm{TM}}$ ) Older Adult Oncology (Version 2.2015). Retrieved from http://www.nccn.org/ professionals/physician_gls/pdf/senior.pdf

Olde Rikkert, M.G., Rigaud, A.S., van Hoeyweghen, R.J., \& de Graaf, J. (2003). Geriatric syndromes: Medical misnomer or progress in geriatrics? Netherlands Journal of Medicine, 61(3), 83-87.

Oncology Nursing Society, \& Geriatric Oncology Consortium (2004). Oncology Nursing Society and Geriatric Oncology Consortium joint position on cancer care in the older adult. European Journal of Cancer Care, 13(5), 434-435.

Oncology Nursing Society, \& Geriatric Oncology Consortium (2007). Oncology Nursing Society and Geriatric Oncology Consortium joint position on cancer care for older adults. [Practice Guideline]. Oncology Nursing Forum, 34(3), 623-624.

Puts, M.T., Papoutsis, A., Springall, E., \& Tourangeau, A.E. (2012). A systematic review of unmet needs of newly diagnosed older cancer patients undergoing active cancer treatment. Supportive Care in Cancer, 20(7), 1377-1394. doi:10.1007/s00520-012-1450-7

Retornaz, F., Monette, J., Batist, G., Monette, M., Sourial, N., Small, D., ... Bergman, H. (2008). Usefulness of frailty markers in the assessment of the health and functional status of older cancer patients referred for chemotherapy: A pilot study. Journals of Gerontology Series A-Biological Sciences \& Medical Sciences, 63(5), 518-522.

Sawhney, R., Sehl, M., \& Naeim, A. (2005). Physiologic aspects of aging: Impact on cancer management and decision making, Part I. Cancer Journal, 11(6), 449-460. 
Sehl, M., Sawhney, R., \& Naeim, A. (2005). Physiologic aspects of aging: Impact on cancer management and decision making, Part II. Cancer Journal, 11(6), 461-473.

Sinding, C., \& Wiernikowski, J. (2009). Treatment decision making and its discontents. Social Work in Health Care, 48(6), 614-634. doi:10.1080/00981380902831303

Statistics Canada (2014). Table 052-0005: Projected population, by projection scenario, age and sex, as of July 1, Canada, provinces and territories, annual (persons). CANSIM [Database]. Retrieved from http://www5.statcan.gc.ca/cansim

Tariman, J.D. (2009). Half of patients with cancer are older than 65: Do you know how to care for older adults? ONS Connect, 24(12), 8-11.

Tariman, J.D., Berry, D., Cochrane, B., Doorenbos, A., \& Schepp, K. (2012). Physician, patient, and contextual factors affecting treatment decisions in older adults with cancer and models of decision making: A literature review. Oncology Nursing Forum, 39(1), E70-E83. doi:10.1188/12.ONF.E70-E83

Walter, L.C., \& Covinsky, K.E. (2001). Cancer screening in elderly patients. JAMA: The Journal of the American Medical Association, 285(21), 2750-2756.

Wedding, U., \& Stauder, R. (2014). Cancer and ageism. ecancer, 8, 39. doi:10.3332/ecancer.2014.ed39

White, H.K., \& Cohen, H.J. (2006). The older cancer patient. Medical Clinics of North America, 90(5), 967-982. doi:10.1016/j. mcna.2006.05.017

Wildiers, H., Heeren, P., Puts, M., Topinkova, E., Janssen-Heijnen, M.L.G., Extermann, M., ... Hurria, A. (2014). International Society of Geriatric Oncology consensus on geriatric assessment in older patients with cancer. Journal of Clinical Oncology, 32(24), 25952603. doi:10.1200/jco.2013.54.8347 